channel blocker vs diuretic: The Antihypertensive and Lipid-Lowering Treatment to Prevent Heart Attack Trial (ALLHAT). JAMA 2002;288:2981-97; erratum 289:178.

14 Wing LM, Reid CM, Ryan P, Beilin LJ et al. A comparison of outcomes with angiotensinconverting-enzyme inhibitors and diuretics for hypertension in the elderly. $N$ Engl J Med 2003;348:583-92.

15 Moser M. Current recommendations for the treatment of hypertension: are they still valid? J Hypertens 2002;20 Suppl 1:S3-10.

$16 \mathrm{MRC/BHF}$ Heart Protection Study of cholesterol lowering with simvastatin in 20,536 high-risk individuals: a randomised placebo-controlled trial. Heart Protection Study Collaborative Group. Lancet 2002; 360:7-22.

17 Montgomery SA. Late-life depression: rationalizing pharmacological treatment options. Review. Gerontology 2002;48: 392-400.

18 Drugs for disruptive features in dementia. Review. Drug Ther Bull 2003;41:1-4.

19 Royal College of Physicians and Bone and Tooth Society of Great Britain. Osteoporosis - clinical guidelines for prevention and treatment. Update on pharmacological interventions and an algorithm for management. London: RCP, 1999, 2000.

20 Bone \& Tooth Society, National Osteoporosis society, Royal College of Physicians. Glucocorticoid-induced osteoporosis: guidelines for prevention and treatment. London: RCP, 2002.
Delirium

Kaiser Nayeem MRCPI, Registrar in Geriatric Medicine

Shaun T O'Keeffe MD FRCPI, Consultant Physician

Department of Geriatric Medicine, Merlin Park Regional Hospital, Galway, Ireland

Clin Med 2003;3:412-5

Delirium (acute confusional state) is a common and unpleasant condition in older people that can have serious short- and long-term consequences. It is often misdiagnosed or unrecognised by doctors and nurses, and management is often poor. ${ }^{1}$

\section{Clinical features and diagnosis}

A diagnosis of delirium should be considered when a patient is described as, or thought to be, 'confused', 'vague', 'a poor historian' or 'unco-operative'.

Delirium is characterised by a change of cognition that develops over hours or days (Table 1). Symptoms fluctuate throughout the day and are worst at night. Disturbed consciousness and inability to attend to the environment are cardinal features: patients are highly

Table 1. Diagnostic criteria for delirium.

- Disturbance of consciousness (reduced clarity of awareness of the environment) with reduced ability to focus, sustain or shift attention

- A change in cognition (such as memory deficit, disorientation, language disturbance or perceptual disturbance) not better explained by a pre-existing or evolving dementia

- The disturbance develops over a short period of time (usually hours to days) and tends to fluctuate over the course of the day

- There is often evidence from the history, physical examination or laboratory findings that the disturbance is due to one or more medications or general medical conditions distractable and find it difficult to focus or sustain concentration. They are often disoriented with rambling, incoherent speech and may be tearful or anxious. Persecutory delusions and visual hallucinations are common.

Two distinct clinical subtypes of delirium are recognised:

- an agitated variant with psychomotor overactivity, such as plucking at bedclothes or aggression, and

- a quiet variant where patients appear apathetic and withdrawn; this is easily missed or misdiagnosed as depression.

A history from a carer of the onset of the cognitive disturbance is invaluable in distinguishing between dementia and delirium. Delirious patients can often be recognised at the bedside from their characteristic distractability. Impaired attentiveness can be assessed formally with bedside tests such as asking the patient to say the months of the year backwards or to count backwards from 20 .

Generalised slowing of the EEG trace is characteristic of delirium (withdrawal states excepted), but the specificity of this finding is reduced with increasing age and in dementia.

The prevalence and incidence of delirium are shown in Table 2.

\section{Outcome}

Delirium is traditionally regarded as a transient disorder, but $30-60 \%$ of delirious patients still have clinically significant new cognitive impairment several weeks later, and subsequently there is an increased risk of developing dementia. ${ }^{4}$ It remains uncertain whether delirium is simply a marker for reduced cognitive reserve or whether it may of itself cause structural brain damage.

In reports from widely diverse healthcare systems, even after adjusting for potential confounding factors, delirium has consistently been associated with: ${ }^{5}$

- prolonged hospital stay

- functional decline, and

- increased risk of institutionalisation.

Complications such as pressure sores, falls, infections and urinary incontinence 
are more common in delirious patients and may prolong or exacerbate the delirium.

\section{Aetiology}

In the past, it was usual to describe the acute illness that led to the patient's admission as the 'cause' of delirium. However, many episodes of delirium develop only after admission to hospital (Table 2) despite the institution of effective therapy for the presenting illness. A better approach is to distinguish between factors that increase the vulnerability of the patient to delirium and those that precipitate delirium. ${ }^{6}$ The precipitating insult in vulnerable patients may be as mild as a simple urinary tract infection. Delirium is almost invariably multifactorial in older people, and it is often inappropriate to isolate a single precipitant as 'the' cause.

\section{Predisposing factors}

The most important risk factors for delirium have consistently emerged as:

- prior cognitive impairment

- older age

- severity of illness, and

- psychoactive drug use.

Laboratory indices of dehydration or metabolic disturbance, alcohol abuse and visual impairment have also been identified as important risk factors in some studies. ${ }^{1}$

\section{Precipitating factors (Table 3)}

Delirium may be the presenting feature of an otherwise clinically silent myocardial infarction or pneumonia. Common precipitants of delirium in hospital patients include:

- chest or urinary infections

- cardiorespiratory disease

- medication toxicity

- dehydration, and

- stroke (especially involving the right hemisphere).

Alcohol withdrawal should always be considered, even in the absence of a history of alcohol abuse. Thiamine defi-

Table 2. Prevalence of and incidence of delirium.

- $10-20 \%$ of older medical patients are delirious on hospital admission

- a further $10-30 \%$ develop delirium after admission ${ }^{2}$

Delirium develops in:

- $5-10 \%$ of patients following general surgery

- $33-50 \%$ of patients after hip fracture repair

- $20-40 \%$ of patients after elective joint replacement surgery

Postoperative delirium is common:

- after major cardiac surgery

- in patients admitted to intensive care ${ }^{3}$

Delirium occurs:

- in up to $60 \%$ of patients admitted to palliative care units

ciency, non-convulsive seizures, intracranial infections and subdural haematoma should be considered in difficult cases. Anticholinergic drugs are important in the genesis of delirium, and metabolites of drugs not usually thought of as having major anticholinergic effects such as digoxin, nifedipine and prednisolone may contribute to the anticholinergic 'burden.' Iatrogenic factors, such as the use of restraints, malnutrition, polypharmacy and bladder catheterisation, are especially important in patients who develop delirium after hospital admission. ${ }^{6}$

\section{Pathophysiology}

It appears that delirium represents the clinical manifestation of diffuse, reversible impairment of cerebral oxidative metabolism and neurotransmission. ${ }^{3}$ The current dominant theory is that a state of relative cholinergic

Table 3. Selected common precipitating causes of delirium.

Medications

Infections

Metabolic abnormalities

Hypoxaemia

Urinary and faecal retention

Pain

Neurological illness
Alcohol or sedative hypnotic withdrawal

Substance toxicity

Sedative hypnotics

Opioids (especially postoperative)

Anticholinergics

Antiparkinsonian drugs

Antidepressants

Anticonvulsants

Corticosteroids

Digoxin

Pneumonia

Urinary tract infections

Hypoglycaemia

Hypercalcaemia

Hypernatraemia

Hepatic failure

Renal failure

Thiamine deficiency

Cardiac failure

Myocardial infarction

Respiratory failure

Pulmonary embolus

Stroke

Seizures

Subdural haematoma 
deficiency and dopaminergic excess is the final common pathway for delirium, although disturbances in other neurotransmitter systems also occur. Another hypothesis is that delirium results from a stress reaction to acute illness, associated with increased levels of and vulnerability to glucocorticoids and cytokines.

\section{Treatment}

The general principles of treatment of delirium are: ${ }^{8,9}$

- early diagnosis

- treatment of precipitating factors

- provision of a supportive environment

- maintenance of nutrition and hydration, and

- careful use (if at all) of psychotropic drugs.

\section{Supportive measures}

It is important to maintain fluid intake and nutrition. Subcutaneous fluids are a useful option in agitated patients. Multivitamin supplements, especially thiamine, are essential in obviously malnourished or alcoholic patients. Physical restraints, including bedrails, are potentially dangerous in paranoid and agitated patients. Spectacles and hearing aids should be provided to minimise sensory deprivation, and self-care and mobility promoted. Interventions such as bladder catheterisation should be avoided unless essential. Staff should adopt a calm and friendly approach, with frequent reassurance. The presence of family members at the bedside will often improve orientation, reduce agitation and facilitate feeding.

These measures represent a humane approach to dealing with the confused patient. However, randomised trials have found that systematic detection and management of delirium lead to statistically significant, but small, beneficial effects on cognition, behaviour and function without affecting long-term outcomes. ${ }^{10}$ In contrast, some similar interventions have been found to be effective in the prevention of delirium in vulnerable patients.

\section{Table 4. Pharmacological management of delirium.}

General principles $\begin{aligned} & \text { Tailor doses according to age, body size, sex and degree of } \\ & \text { agitation }\end{aligned}$
- Titrate doses to effect
- Increase scheduled dose if regular 'as-needed' doses required
Haloperidol
-

Atypical neuroleptic - Fewer drug interactions and less extrapyramidal effects than haloperidol

- ? Similar effects on QTC

- Antipsychotic potency: risperidone $>$ olanzapine $>$ quetiapine

- Sedation: olanzapine $>$ quetiapine $>$ risperidone

- Extrapyramidal safety: quetiapine $>$ olanazapine $>$ risperidone

- Usual starting doses:

- risperidone $0.5 \mathrm{mg} \mathrm{bd}$

- olanzapine $2.5-5.0 \mathrm{mg}$ daily

- quetiapine 25-50 mg daily

$\begin{array}{ll}\text { Lorazepam } & \text { Benzodiazepine } \\ \text { - } & \text { Use in sedative and alcohol withdrawal, parkinsonism } \\ \text { extrapyramidal side effects } & \text { More likely than neuroleptics to cause respiratory depression and } \\ \text { paradoxical excitement }\end{array}$

\section{Pharmacological management}

Not all patients need psychotropic medications. While they may treat troublesome symptoms, their effect on long-term outcomes is unknown. The need for medication is usually obvious in the agitated, hallucinating patient but even quietly delirious patients can experience considerable distress.

The common errors in managing delirium are to use antipsychotic medications in excessive doses, give them too late and to overuse benzodiazepines. It is kinder and less dangerous to begin regular low-dose therapy early rather than wait for agitation to increase and then resort to a 'chemical cosh'. The only randomised trial to date, albeit in younger AIDS patients, has confirmed that neuroleptic agents are superior to benzodiazepines in the treatment of delirium. ${ }^{11}$ Neuroleptics do not impair respiratory function and are less likely than benzodiazepines to cause drowsiness or disinhibition.

Neuroleptics. Haloperidol remains the standard treatment; it is a powerful antipsychotic that can be given orally or parenterally and has limited anticholinergic, sedative, hypotensive or 


\section{Key Points}

Delirium in older patients is usually multifactorial in origin, and there is an inverse relationship between the severity of the insult necessary to precipitate delirium and the pre-existing vulnerability of the patient

Delirium associated with impaired cholinergic neurotransmission is contributed to by metabolites of drugs not usually thought of as having major anticholinergic effects

The cognitive effects of delirium may resolve only slowly or not at all

Neuroleptic agents appear to be superior to benzodiazepines in the pharmacological treatment of delirium

Measures to promote orientation and sleep and to avoid sensory deprivation and dehydration can prevent delirium in high-risk patients

KEY WORDS: agitation, cognitive impairment, delirium, haloperidol, lorazepam, pharmacotherapy

pro-arrhythmic properties (Table 4) ${ }^{8,12}$ An initial, regular dose of $0.5-1.0 \mathrm{mg}$ haloperidol twice daily will often suffice in older people, with additional as-needed doses four-hourly.

Although there are few trial data, atypical neuroleptics also seem effective and are less likely to cause extrapyramidal side effects. Of these agents, risperidone has the greatest antipsychotic potency, olanzapine is most likely to cause sedation, and quetiapine is least likely to cause parkinsonism.

The response to treatment should be monitored daily; if as-needed medication is needed regularly, the dose should be increased. When patients are stable, antipsychotics should be continued for a few days, then tapered and stopped.

Prolongation of the rate-corrected QT interval (QTc) can occur with neuroleptics, especially at high doses or if there is concomitant electrolyte disturbance, heart disease or use of other QTc prolonging drugs. Prolonged QTc may increase the risk of developing torsades de pointes and sudden death, although the size of the risk with currently favoured neuroleptics is unknown. Baseline electrolyte measurement and ECG seem prudent in high-risk patients. The American Psychiatric Association suggests that cardiac monitoring or discontinuation or reduction in neuroleptic dose should be considered with QTc prolongation greater than $450 \mathrm{msec}$ or more than $25 \%$ increase above baseline. ${ }^{9}$
Other agents. Benzodiazepines are the treatment of choice for withdrawal states and for patients with extrapyramidal disease. Lorazepam, which has a relatively short half-life and is available for parenteral use, is particularly useful. The antidepressant trazadone is a useful alternative to benzodiazepines in patients with parkinsonism and delirium, although sedative side effects can occur.

Trials are investigating whether cholinesterase inhibitors such as donepezil may be useful in treating (or preventing) delirium. Psychostimulants have been used to treat hypoactive delirium, but there have been no satisfactory studies.

\section{Prevention}

There is now good evidence that delirium in high-risk patients can be prevented by a strategy comprising:

- repeated reassurance and orientation of the patient

- non-pharmacological sleep promotion

- early mobilisation

- provision of visual and hearing aids, and

- avoidance of dehydration.

This strategy reduced the incidence and duration of delirium in a study of 852 elderly patients on acute medical wards. The number of patients needed-to-treat to prevent one episode of delirium was 19 (95\% confidence interval 10-134). ${ }^{13}$

\section{References}

1 Lindesay J, Rockwood K, Macdonald A (eds). Delirium in old age. Oxford: Oxford University Press, 2002.

2 Levkoff SE, Evans DA, Liptzin B, Clearey PD et al. Delirium. The occurrence and persistence of symptoms among elderly hospitalized patients. Arch Intern Med 1992;152: 334-40.

3 O'Keeffe ST, Ni Chonchubhair A. Postoperative delirium in the elderly. Review. Br J Anaesth 1994;73:673-87.

4 Rockwood K, Cosway S, Carver D, Jarrett P et al. The risk of dementia and death following delirium. Age Ageing 1999;28: 551-6.

5 Inouye SK. The dilemma of delirium: clinical and research controversies regarding diagnosis and evaluation of delirium in hospitalized elderly medical patients. Am J Med 1994;97:278-88.

6 Inouye SK, Charpentier PA. Precipitating factors for delirium in hospitalized elderly persons. Predictive model and interrelationship with baseline vulnerability. JAMA 1996;275:852-7.

7 Tune L, Carr S, Hoag E, Cooper T. Anticholinergic effects of drugs commonly prescribed for the elderly: potential means of assessing risk of delirium. Am J Psychiatry 1992;149:1393-4.

8 Meaghar DJ. Delirium: optimising management. Review. BMJ 2001;322:144-9.

9 Practice guideline for the treatment of patients with delirium. American Psychiatric Association. Am J Psychiatry 1999;156(5 Suppl 1):1-20.

10 Cole MG, Primeau F, McCusker J. Effectiveness of interventions to prevent delirium in hospitalized patients: a systematic review. CMAJ 1996;155:1263-8.

11 Breitbart W, Marotta R, Platt MM, Weisman $\mathrm{H}$ et al. A double-blind trial of haloperidol, chlorpromazine, and lorazepam in the treatment of delirium in hospitalised AIDS patients. Am J Psychiatry 1996;153:231-7.

12 Taylor D, Lewis S. Delirium. Review. J Neurol Neurosurg Psychiatry 1993;56: 742-51.

13 Inouye SK, Bogardus ST Jr, Charpentier PA, Leo-Summers L et al. A multicomponent intervention to prevent delirium in hospitalized older patients. N Engl J Med 1999; 340:669-76. 